\title{
The Search for Life as a Guidepost to Scientific Revolution
}

\author{
Authors \\ Casey Dreier \\ The Planetary Society \\ casey.dreier@planetary.org \\ 626-793-5100 \\ Bill Nye \\ The Planetary Society \\ Co-Signed By \\ The Planetary Society \\ Board of Directors
}




\section{Summary}

The planetary science community should embrace the search for life as a prime organizing science principle of the decadal survey. The discovery of life beyond Earth, particularly in the form of extant life within the solar system, would be a discovery of such significance that it would be a discontinuity in human knowledge — a scientific revolution.

The National Academies' Astrobiology Strategy (National Academies 2019) outlined practical steps that the planetary community should follow to pursue this goal. The planetary community should also prioritize in-situ and, ultimately, sample return missions focused on the habitable environments in our solar system, and work closely with the astronomy community on the detection of habitable exoplanets.

Ambitious mission goals need not require ambitious mission budgets. A comprehensive search for life must find ways to diversify its efforts beyond flagship missions into mid- and small-class programs in order to increase mission cadence and thus the potential for biosignature detection.

The search for life in our solar system presents a unique opportunity, if embraced by the planetary science community, to implement a clear, encompassing strategy that integrates multiple scientific disciplines and solar system destinations. If life exists to be discovered, the outcome would be, at minimum, historic and, at maximum, revolutionary to the applied sciences of biology and medicine. We have the capability. We know where to look. The time is right.

\section{Finding Life is a Scientific Revolution Waiting to Happen}

The scientific revolution that began in the 16th century ${ }^{1}$ coincided with the application of natural laws to the cosmos. Initially, astronomy confirmed that the physics of motion worked the same in the heavens as it did on Earth. Later on, spectral analysis extended the science of chemistry to stars and other celestial phenomena. In the mid-20th century, the exploration of our solar system established that the science of geology applies to worlds beyond our own. Here in the early decades of the 21st century, we have the opportunity to test if the science of biology similarly extends beyond Earth (Hays 2015, Hendrix and Hurford 2019, Onstott 2019, Hand 2020).

The confirmation that biology "works" beyond Earth, either by the detection of biosignatures or, more importantly, the discovery of extant life, would be so significant that it represents a functional discontinuity in human knowledge (Figure 1). Discontinuities such as these can be considered scientific revolutions.

\footnotetext{
${ }^{1}$ Obviously, the exact timing and nature of the "scientific revolution" is debated among scholars. For the purposes of this paper, we use the 1543 publication of De revolutionibus orbium coelestium by Copernicus as our anchor point.
} 


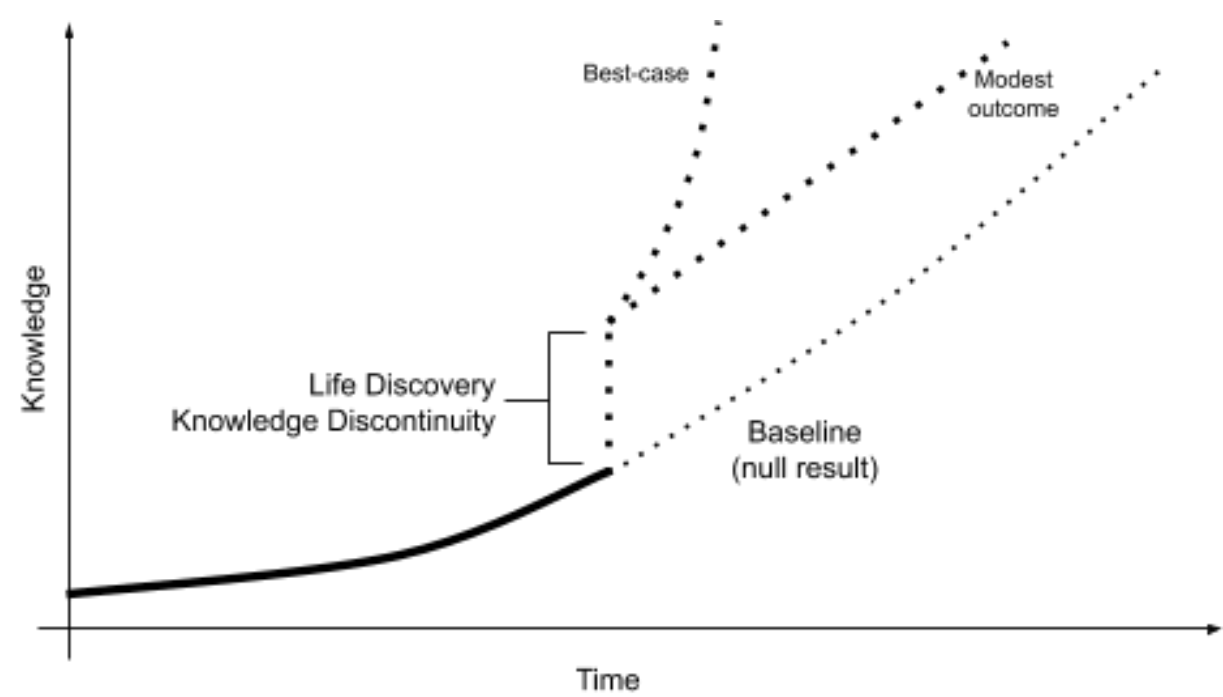

Figure 1. The discovery of life would provide such a rapid influx of new knowledge that it can be considered a "discontinuity" in the knowledge of biological sciences, making it difficult to predict whether the consequences would be significant (exponential knowledge growth) or modest (linear trend line). Either way, the knowledge of the field would be greater than a null result.

As with any revolution, its consequences are impossible to predict in advance. We can, however, consider a range of outcomes.

Assuming life is out there in the first place, its discovery could yield only temporary public interest and a new academic subfield of exobiology. While this would be undeniably a positive outcome, given that scientific knowledge has inherent value (Schwartz and Dreier 2020), its benefits would remain abstract to most individuals on Earth. Call this the "modest" outcome.

On the other hand, the discovery of life may provide unique and previously unimaginable insights into structural, chemical, and metabolic aspects of biological systems. These insights could spur subsequent revolutions in applied biology and medicine, leading to improved human well-being and reductions in physical suffering. The discovery could also trigger a social-political awakening that Earth is one of many biological systems, reconfiguring humanity's relationship to other forms of life and to itself - a super-charged version of Apollo 8's "Earthrise" photo that helped spark the environmental movement in the 1970s (Poole 2008). Consider this the "best-case" scenario.

Of course, there is also the very real possibility of a null result - that there is no life to be discovered or that it is simply too difficult to detect. But over the past 50 years, astrobiology research shifted our understanding of the nature of life from a randomly initiated process (i.e. the Miller-Urey experiment) to an emergent physical phenomenon, derived from — or at the very least dependent upon - the planet on which it took hold. The search for life now relies on context that, regardless of positive detections, provides important science and deepens our understanding 
of planetary processes and life itself. In that outcome, human knowledge would continue to grow as a continuous function of research input and global investment.

Given the massive benefits of the "best-case" scenario and the negligible downsides of null results, are we not compelled to pursue this unlikely, potentially game-changing, opportunity?

\section{The Search for Life Provides a Guidepost to Scientific Revolution}

Whereas many avenues of research could result in scientific revolutions, few have such clear guideposts for achieving that condition. The search for life unusually generous in that regard.

We know where to look. The initial robotic reconnaissance of the solar system, with additional insight from the exploration of Earth's deep ocean trenches and other extreme environments, identified habitable oceans in the outer solar system on Europa and Enceladus. Mars may support a biosphere in its subsurface, and, should life have flourished during its ancient periods of habitability, has accessible river deltas, lake basins, and hydrothermal deposits that could have collected and concentrated evidence of its past existence. Further afield, the Kepler and TESS missions have discovered dozens of theoretically habitable planets.

We know how to look, or at least how to start. As the science of the origin of life on Earth has matured, so too has our ability to focus investigations in potential origins of life on other worlds. The discovery of deep ocean vents with alkaline chemistry and porous, organic-concentrating structures provide intriguing hints to the evolution of cells (Martin and Russel 2006) and have potential analogs in ocean worlds in the solar system. Advances in statistical analysis of biological systems provide means to seek biosignatures via in-situ sample analysis (Davila and McKay 2014, Marshall 2017, Johnson 2018).

Perhaps more to the point: the first steps are already happening. Mars Perseverance will perform in-situ analysis and prepare samples of the Jezero crater delta area for Earth return. The Europa Clipper will, for the first time, characterize the Europan ocean and ice shell, potentially flying through the moon's intermittent water plumes in the process. Exoplanets, including those in habitable zones, are being discovered by ground- and space-based telescopes. But these are only the first steps. Sample return and in-situ missions must follow Perseverance at Mars, and Europa Clipper will not directly analyze the surface ice, much less the ocean.

The National Academies' astrobiology strategy provides a set of well-considered recommendations for pursuing a comprehensive, cross-disciplinary effort to search for life, which we will not repeat here. We do note, however, that the report identified no "miracles" necessary in either theory or in technology in order to begin this work. The potential for discovery, then, is a function of how intensely the search is pursued. 


\section{The Practical Benefits of the Search for Life}

"Searching for life" is a clear, accessible statement of priority that is easily communicated with the public and with policymakers. The value of this should not be discounted. "If you're explaining, you're losing" is an old political axiom that emphasizes the importance of clarity and accessibility. The importance, excitement, and profundity of "searching for life" can be immediately understood by most any person, though it certainly benefits from a clarifying discussion about what "life" is and what type is likely to exist in our solar system.

Additionally, the effort of searching for life returns important data for a variety of aspects of planetary science. Even the failure of a search, a null result, provides data for how likely it is that life can form, and can potentially reveal new insights into the origins of life on Earth. If the emergence of life is a "planetary phenomenon", then important planetary science discoveries will be made in the course of such a search.

\section{Would Non-Detection Hurt Planetary Science?}

A critique is that NASA went big on the search for life in the 1970s with the Viking missions, which, after they failed to confirm life, contributed to a collapse in Mars exploration for a generation.

The reality is more nuanced. While it's true that there was a 17-year gap between the launch of the Vikings and of Mars Observer, there were decades-long gaps for every other solar system destination as well. The entire field went through a "survival crisis" for the better part of a decade (Logsdon 2013).

Success or failure is also about the scope of the investigation-Viking proceeded with little context, but the lessons learned from the mission transformed our understanding of the environment on Mars and lit the fire under a now well-established field of research that has come of age in the past 50 years.

Viking was also a programmatic anomaly. Adjusted for inflation it remains, by far, the most expensive planetary mission ever attempted. It was not part of any program line or long-term strategy. Its development cost overshadowed a collapse in other planetary science funding at the time. The final Mariner mission launched 2 years before Viking. The Pioneer line had but a single mission left. Every lunar program had ended by 1968. Absent a brief uptick to support Voyager, the remaining planetary exploration program budget declined throughout the 1970s 
(Figure 2). This prevented new program starts, a problem that became apparent in the 1980s as planetary efforts withered to a single development mission. ${ }^{2}$

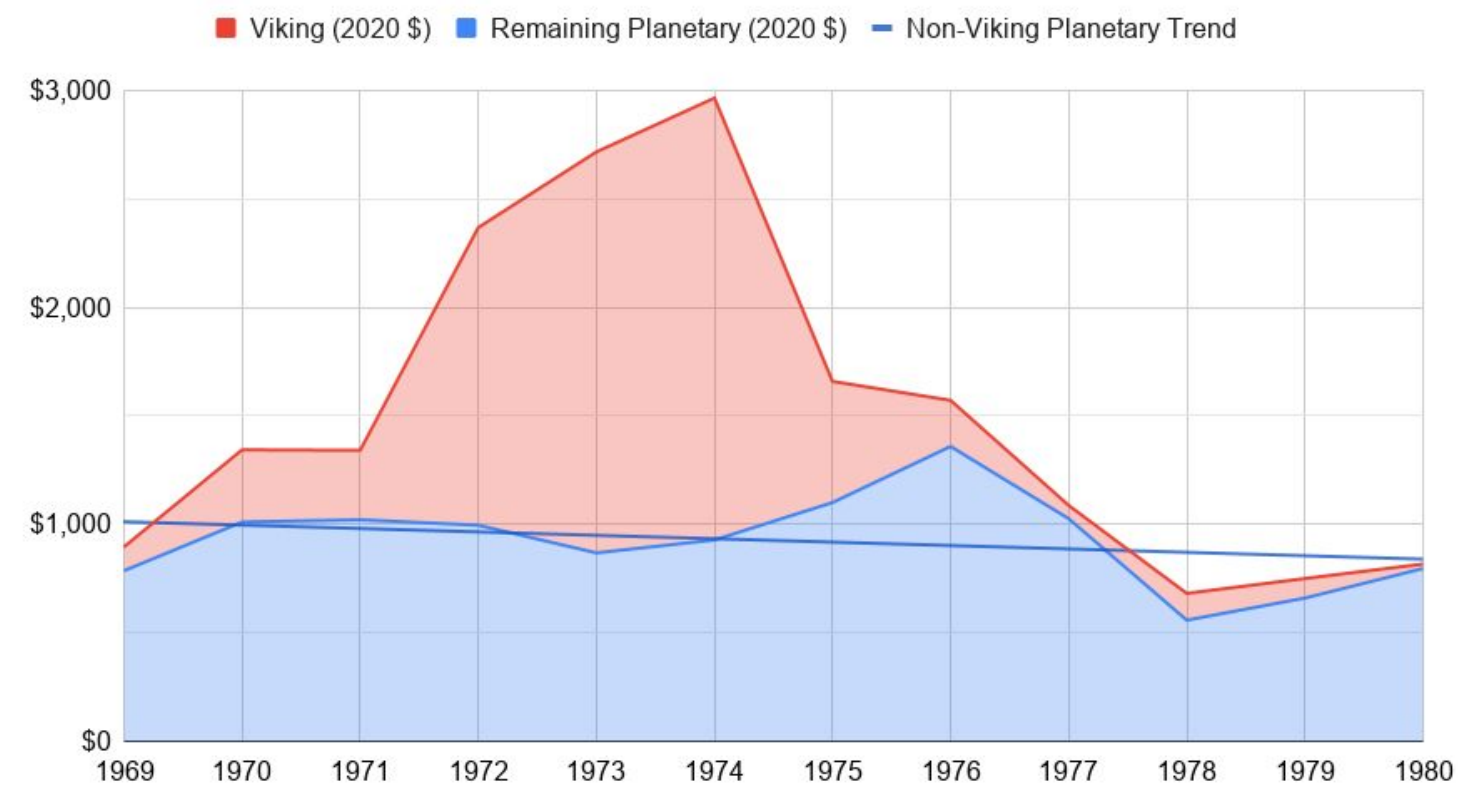

Figure 2. NASA's planetary science funding, with funding for project Viking broken out separately to show the "anomaly" compared to the overall trend of planetary science in the 1970s (Dreier 2020). Dollar amounts are adjusted for inflation via NASA's New Start Index. Vertical axis is millions of dollars. Horizontal axis is fiscal year.

During this period, NASA lacked clear direction in its robotic planetary exploration program. This lack of programmatic clarity fed the belief that planetary science had little more to contribute which, in turn, made it difficult to gain additional political support and funding. While there can be a risk in pursuing ambitious goals, there are similar risks and consequences for thinking small—do so and the policy apparatus will treat one's priorities accordingly.

\section{Synergies With Other Sciences}

The National Academies' Astrobiology Strategy outlines significant and rich opportunities for a multi-disciplinary effort toward the search for life, including new space telescopes, the exploration of subsurface Mars and ocean worlds, research on biosignatures, and technology maturation of life-detection instrumentation. The Planetary Society recommended that the astrophysics community "think big" and pursue large aperture space telescopes with the capability to discern statistically-significant biosignatures in exoplanetary atmospheres (Dreier 2019). Planetary science can serve a complementary role to astrophysics, which is currently developing two major space telescopes (JWST and RST) that will further the search for biosignatures in the electromagnetic spectrum. Planetary exploration can and should search in-situ for life in our own cosmic backyard.

\footnotetext{
${ }^{2}$ Galileo was NASA's only planetary mission in active development from fiscal years 1979 through 1983.
} 


\section{Considerations for the Decadal Committee}

Given that the search for life provides a clear organizing principle for planetary exploration, that it provides a rare guidepost to scientific revolution, and that a potential discovery could significantly improve the well-being of humanity, we believe the decadal committee should consider the following:

- Embrace the search for life as a prime organizing science principle of the decadal survey. The community should go big on the effort.

- The most important goal should be to increase the rate of potential biosignature detection. Flagship-only projects would limit mission selection rate. Medium- and small-class missions can focus on potential biosignature detections which can be followed up by comprehensive attempts to confirm promising signals.

- In addition to mission development, there must be a corresponding priority increase in fundamental research and biosignature-detection technology development.

- The planetary community should work with all NASA science divisions, particularly astrophysics, to pursue a coordinated, complementary effort.

\section{Conclusion: Going Big on the Search for Life}

Searching for life is a challenge. Life may prove elusive to detect. There may be no life to find at all. A biosignature detection may not be definitive or may be merely suggestive. A discovery of microbial life may be of passing interest to a public that, through popular culture, is primed to expect intelligent civilizations or complex, multicellular organisms.

Nonetheless, the benefits far outweigh the potential downsides. The best-case scenario is nothing short of a scientific revolution with broad applications to biology, medicine, and the human relationship to other life on Earth. The search for life is a process of scientific discovery that explores the full scope of planetary evolution. The vast good of the potential positive outcome, when weighed against modest the investment it requires, overwhelms any downsides of failure.

The search for life provides immediate, practical benefits as well. It gives a clear and accessible means by which to communicate major goals of planetary exploration. It enables other scientific investigations. It is complementary to work in astrophysics and origin of life studies on Earth. Even a null result tells us something about the planet and the likelihood of life in the solar system and beyond.

The potential for discovery, then, is a function of how intensely the search is pursued. We will not find life if we do not look for it. The path to a scientific revolution in our understanding of the cosmos is clear. It's time to embrace it. 


\section{References}

Davila, A.F. and C.P. McKay. (2014). Chance and Necessity in Biochemistry: Implications for the Search for Extraterrestrial Biomarkers in Earth-like Environments. Astrobiology. 534-540.

Dreier, C. (2020). Planetary Exploration Budget Dataset. The Planetary Society

Dreier, C; Hammel, H; Ehlmann B; and J. Bell. (2019). Thinking Big: How Large Aperture Space Telescopes Can Aid the Search for Life in Our Lifetimes. Astro2020: Decadal Survey on Astronomy and Astrophysics, science white papers, no. 377.

Hand, K. (2020). Alien Oceans: The Search for Life in the Depths of Space. Princeton University Press.

Hays, L. et. al. (2015). NASA Astrobiology Strategy. NASA.

Hendrix, A. and Hurford, T., et. al. (2019). The NASA Roadmap to Ocean Worlds. Astrobiology.

Logsdon, J. (2013). Survival Crisis of the US Solar System Exploration Program in the 1980s. In: Exploring the Solar System: The History and Science of Planetary Exploration. Roger, L., ed. Palgrave Macmillan.

Martin, W. and M.J. Russel. (2006). On the origin of biochemistry at an alkaline hydrothermal vent. Phil. Trans. R. Soc. B3621887-1926.

Marshall, S.M. et. al. (2017). A probabilistic framework for identifying biosignatures using Pathway Complexity. Trans. R. Soc. A.

Johnson, S., et. al. (2018). Fingerprinting Non-Terran Life. Astrobiology.

National Academies of Sciences, Engineering, and Medicine. (2019). An Astrobiology Strategy for the Search for Life in the Universe. Washington, DC: The National Academies Press.

Onstott, T.C., et. al. (2019). Paleo-Rock-Hosted Life on Earth and the Search on Mars: a Review and Strategy for Exploration. Astrobiology.

Schwartz, J. and C. Dreier (2020). Our Ethical Obligation to Planetary Science in the Age of Competitive Space Exploration. Submission to the Planetary Science Decadal Survey.

Poole, R. (2008). Earthrise: How Man First Saw the Earth. Yale University Press. 